\title{
Evaluation of PSEN1 subunit of the $\gamma$-secretase gene in patients with psoriasis vulgaris: a pilot study
}

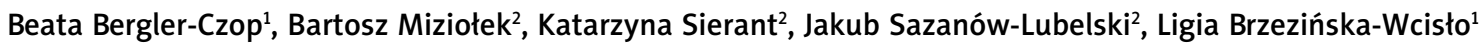

${ }^{1}$ Department of Dermatology, Medical University of Silesia, Katowice, Poland

${ }^{2}$ Chair and Department of Dermatology, Andrzej Mielęcki Silesian Independent Public Hospital, Katowice, Poland

Adv Dermatol Allergol 2020; XXXVII (6): 915-920

DOI: https://doi.org/10.5114/ada.2020.102108

\begin{abstract}
Introduction: Psoriasis is a chronic autoimmune inflammatory disease, the prevalence of which is $1-3 \%$ in the Polish population. Genome testing using single nucleotide polymorphisms revealed more than 50 regions associated with the risk of psoriasis, and most of these genes are associated with the immune system.

Aim: To assess the presence of PSEN1 subunits of the $\gamma$-secretase gene polymorphisms in patients with psoriasis and comparison of results with a healthy control group.

Material and methods: We used polymerase chain reaction - restriction fragment length polymorphism (PCR RFLP) method to assess polymorphisms. The starting material for analysis was peripheral blood obtained from the patient. Results: PSEN1a-positivity was found in 2/52 (2.78\%) of patients with psoriasis and 1/36 (3.85\%) of healthy controls. PSEN1b positivity was seen in $3 / 52$ (5.77\%) of patients with psoriasis and $1 / 36(3.85 \%)$ of control individuals. Only 3 patients with psoriasis but none of healthy volunteers had a presence of PSEN1c. Four patients were excluded from further statistical analysis.

Conclusions: We have not shown a relationship between PSEN1 polymorphism and the clinical occurrence of psoriasis but now we start the assessment of other subunits of the $\gamma$-secretase gene - PSENEN and NCSTN.
\end{abstract}

Key words: $\gamma$-secretase, PSEN1, psoriasis vulgaris.

\section{Introduction}

Psoriasis is a chronic autoimmune inflammatory disease, the prevalence of which is $1-3 \%$ in the Polish population. It is characterized by excessive proliferation of keratinocytes, a disorder of their differentiation as well as infiltration of inflammatory cells within the skin [1]. It is clinically manifested by various sizes of papules or plaques covered with silvery scales, most often on the skin around the knees, elbows, loins and scalp. Its aetiology has not been fully understood and is still a great challenge to researchers. It is known that immune disorders, environmental factors (including infections, injuries, emotional stress, certain medications) and genetic background play an important role [2]. Ozawa et al. [3] showed a statistically significant high incidence of HLACW1 and HLA-DQB1*0303 alleles among Japanese patients suffering from non-pustular and pustular psoriasis as well as a high incidence of HLA-B46 alleles in Japanese patients with pustular psoriasis and a previous history of non-pustular psoriasis. The risk of developing psoriasis in monozygotic twins is $70 \%$, while for dizygotic twins is $20 \%$ [2]. Genome testing using single nucleotide polymorphisms revealed more than 50 regions associated with the risk of psoriasis, and most of these genes are associated with the immune system [1, 2].

Genetic linkage studies of multiply affected families have mapped susceptibility loci to chromosomes 1q21, 3q21, 4qter, 6p21.3, 14q31, 17q24-25, and 19p13 [4, 5]. The isolation of novel susceptibility genes will provide insights into the precise biochemical pathways that control this disease. Ruissen et al. [6] carried out serial analysis of gene expression (SAGE) of purified keratinocytes derived from skin biopsy specimens. They identified 7645 unique SAGE tags from epidermal cells. Zhou et al. [7] identified 1338 uniquely expressed genes which were thought to have potential roles in the pathogenesis and maintenance for psoriasis. Among those genes, they especially focused on chemokines which may have sig-

Address for correspondence: Assoc. Prof. Beata Bergler-Czop, Department of Dermatology, Medical University of Silesia, 20-24 Francuska St, 40-027 Katowice, Poland, phone/fax: +48 3225611 82, e-mail: bettina2@tlen.pl, bbergler-czop@sum.edu.pl Received: 3.02.2019, accepted: 27.04.2019. 
nificant roles in sustaining T-cell activation and chronic inflammation in psoriatic lesions through dendritic cell effects.

In a study performed by Itoh et al. [8], 241 genes were differentially expressed only in involved psoriatic skin but not in the other samples. They also identified seven genes that were reported to be related to antiproliferation such as $\alpha 1$-microglobulin/bikunin precursor gene (AMBP), leucine zipper, down-regulated in cancer 1 protein (LDOC1), claudin 1 (CLDN1), unc-13-like protein (UNC13B), calnexin (CANX), tyrosinase-related protein 1 (TYRP1), and Yes-associated protein 1, 65 kDa (YAP1). These seven genes, which they reported to be related to apoptosis or antiproliferation, might have causal roles in pathophysiology in psoriasis.

Notch signalling controls a number of cellular processes including cell fate decision, proliferation, differentiation and survival/apoptosis. Hence, Notch and its ligands are expressed in multiple tissues including the skin, where they are abundantly expressed in the epidermis. Notch activation results in the promotion of growth arrest and the onset of differentiation, therefore suggesting that specific Notch activation may regulate skin homeostasis by balancing these processes, i.e. Notch signalling functions as a molecular switch that controls the transition of cells between skin layers during the epidermal differentiation process. Aberrant Notch signalling results in the development of psoriasis and skin cancers such as squamous cell carcinoma, basal cell carcinoma and malignant melanoma [9-11].

Skin disorders are observed in mice with skin-specific inactivation of the Notch1 gene, which encodes another transmembrane protein cleaved by $\gamma$-secretase, suggesting that Notch1 is the enzyme's relevant substrate [12, 13].

Wang et al. [14] reveal that mutations in PSEN1 as well as in the PSENEN and NCSTN genes that encode the
PEN2 and nicastrin subunits of $\gamma$-secretase, respectively, cause acne inversa. This is consistent with mouse studies showing that $\gamma$-secretase deficiency produces follicular hyperkeratosis, an initiating event in acne inversa. Hsu et al. [15] observed exacerbation of psoriatic skin lesions in a patient receiving $\gamma$-secretase inhibitor so we also think about the role of polymorphisms or mutation of subunits of this enzyme in patients with psoriasis vulgaris.

\section{Aim}

The aim of this study was to assess the presence of PSEN1 subunits of the $\gamma$-secretase gene polymorphisms in patients with psoriasis vulgaris and comparison of results with a healthy control group.

\section{Material and methods}

Fifty-two patients with psoriasis vulgaris were included in the study, and the control group consisted of 36 healthy volunteers.

Patients inclusion criteria: age 18-70, diagnosis of psoriasis vulgaris clinically and/or histologically, agreement for the study. Exclusion criteria: amyloidosis, Alzheimer disease, acne inversa.

The starting material for assessing the polymorphism was peripheral blood. DNA extraction as a template for the analysis of polymorphisms was carried out using the DNA purification kit - GeneMATRIX QUICK BLOOD (EURX, Gdansk,Poland, E-3565). Extraction was carried out according to the manufacturer's protocol. The final volume of eluate containing DNA in apyrogenic water was $60 \mu \mathrm{l}$. DNA obtained from peripheral blood was a template for carrying out a PCR reaction, the aim of which was to obtain a product, a fragment of the gene coding for the PSEN1 gamma subunit - secretase. For this purpose, 3 separate PCR reactions were carried out, for each chosen

Table 1. Method of evaluation of the polymorphism

\begin{tabular}{|c|c|c|c|c|}
\hline Gene & Polymorphism & Primers & $\begin{array}{c}\text { Temperature of connecting } \\
\text { the starters }\left[{ }^{\circ} \mathrm{C}\right]\end{array}$ & $\begin{array}{l}\text { Product length } \\
{[\mathrm{pz}]}\end{array}$ \\
\hline \multirow[t]{2}{*}{ PSEN1a } & \multirow{2}{*}{$\begin{array}{l}\text { NT_026437.13 } \\
\text { (rs1800844) }\end{array}$} & F - 5'-TGTCCTTGTCCAGGGGCGGC-3' & \multirow[t]{2}{*}{63.8} & \multirow[t]{2}{*}{182} \\
\hline & & R - 5'-GCAGAGCTGACCACAACGGTGAGAAG-3' & & \\
\hline \multirow[t]{2}{*}{ PSEN1b } & \multirow[t]{2}{*}{ NT_54961 } & F - 5’ AGGAGGGGCGGCCCGTTTCTCG 3' & \multirow[t]{2}{*}{66} & \multirow[t]{2}{*}{520} \\
\hline & & R - 5’ AGCCTCTGCCACCACCGCAGATC 3' & & \\
\hline \multirow[t]{2}{*}{ PSEN1C } & \multirow[t]{2}{*}{ NT_49654 } & F - 5' GGGAGTTTTAAAGGGGTCTAG 3' & \multirow[t]{2}{*}{63} & \multirow[t]{2}{*}{519} \\
\hline & & R - 5' TTGCCTCTGCTGTAAGACAA 3' & & \\
\hline
\end{tabular}

Table 2. Enzymatic digestion using restriction enzymes

\begin{tabular}{lccc}
\hline Gene & Polymorphism & Restriction enzyme & \multicolumn{2}{c}{ Result of restriction enzyme cleavage } \\
\hline PSEN1a & NT_026437.13 (rs1800844) & Haelll (BsuRI) & G: 182; C: 164+18 \\
\hline PSEN1b & NT_54961 & BstXI & G: 520; C: 268+252 \\
\hline PSEN1C & NT_49654 & Mbol & C: 329+190; T: 208+190+121 \\
\hline
\end{tabular}




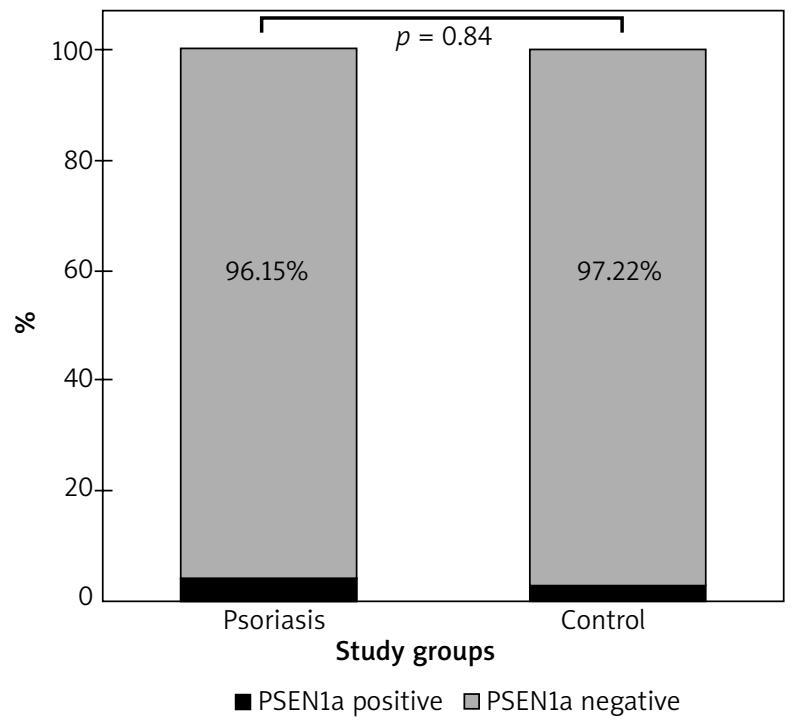

Figure 1. PSEN1a-positivity was found in $2 / 52(2.78 \%)$ of patients with psoriasis and 1/36 (3.85\%) of healthy controls

for polymorphism analysis, using appropriately selected primers (Table 1). The selected primers allowed for the amplification of a selected fragment of the gene in which the polymorphic site is evaluated. The Color Perpetual OptiTaq PCR Master Mix 2x (EURx, Gdansk, Poland E2775-03) was used to carry out the $P C R$ reaction. The $P C R$ reaction mixture contained:

- Color Perpetual OptiTaq PCR Master Mix 2x - $25 \mu$,

- starter forward (10 uM) - $2.5 \mu \mathrm{l}$,

- starter reverse (10 uM) - $2.5 \mu \mathrm{l}$,

- water - $8 \mu \mathrm{l}$,

- DNA matrix - $12 \mu \mathrm{l}$.

The PCR reaction was carried out under the following thermal conditions:

- preliminary denaturation $-94^{\circ} \mathrm{C}-3 \mathrm{~min}$;

-40 cycles: denaturation $-94^{\circ} \mathrm{C}-20 \mathrm{~s}$, joining the starters $-X^{\circ} \mathrm{C}-30 \mathrm{~s}(\mathrm{X}$ - temperature depending on the used primers), product extension $-72^{\circ} \mathrm{C}-60 \mathrm{~s}$;

- final product lengthening: $72^{\circ} \mathrm{C}-7 \mathrm{~min}$.

The result of the PCR reaction was visualized to evaluate the products obtained by using an electrophoresis technique in a $2 \%$ agarose gel. The products were stained by using the SimpleSafe reagent (EURx, Gdansk, Poland E4600-01). Next, the obtained PCR products were subjected to enzymatic digestion using restriction enzymes (FastDigest, Thermo Scientific) used to assess the selected polymorphism (Tables 1, 2).

The result of the enzymatic cleavage of the product was visualized using agarose or polyacrylamide gel electrophoresis technique (depending on the product being evaluated).

$\chi^{2}$ test with Fisher's exact mid-P method was applied to evaluate differences in the prevalence of PSEN(1a-1c)

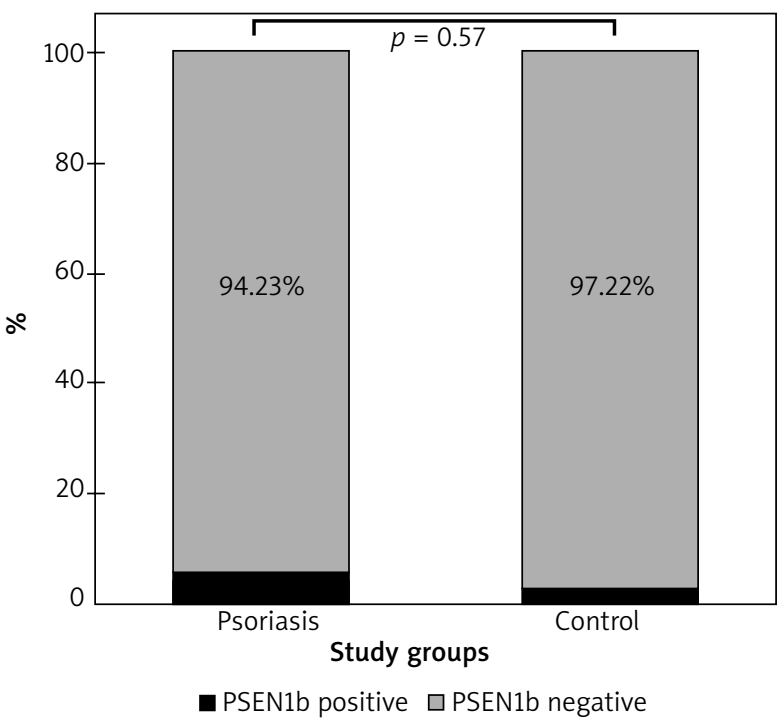

Figure 2. PSEN1b positivity was seen in $3 / 52$ (5.77\%) of patients with psoriasis and 1/36 (3.85\%) of control individuals

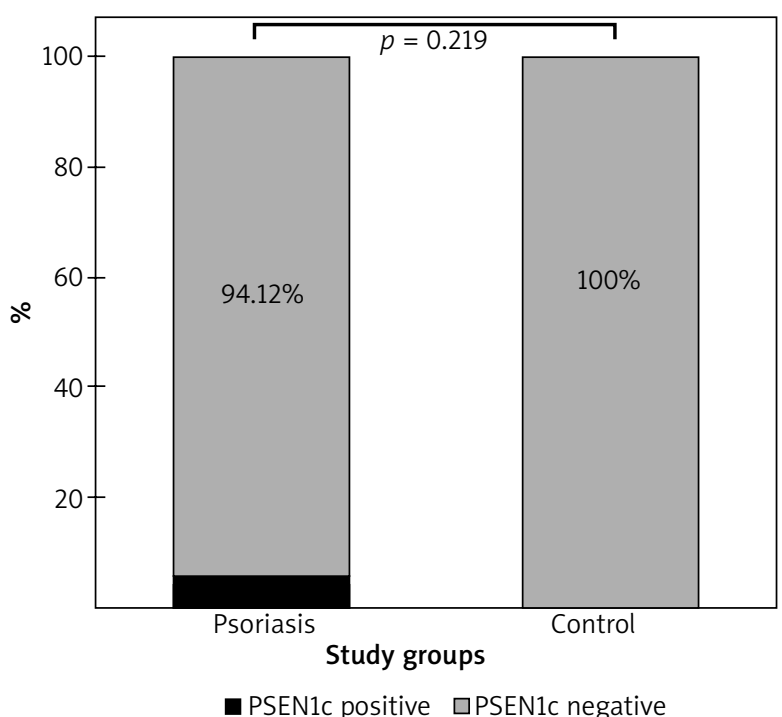

Figure 3. Three patients with psoriasis but none of healthy volunteers had a presence of PSEN1c

polymorphism between psoriatic patients and healthy controls. A value of $p<0.05$ was considered significant.

The study was approved by the regional bioethical committee.

\section{Results}

PSEN1a-positivity was found in 2/52 (2.78\%) of patients with psoriasis and $1 / 36$ (3.85\%) of healthy controls (Figure 1). PSEN1b positivity was seen in 3/52 (5.77\%) of patients with psoriasis and $1 / 36$ (3.85\%) of control individuals (Figure 2). Only 3 patients with psoriasis but none 
Table 3. PSEN 1a, b

\begin{tabular}{ccccc}
\hline \multicolumn{2}{c}{ PSEN1a } & \multicolumn{2}{c}{ PSEN1b } & \multirow{2}{*}{ Total } \\
\cline { 1 - 4 }$+\boldsymbol{n}$ & - & + & - & \\
\cline { 1 - 4 } 2 & 50 & 3 & 49 & 52 \\
\hline 1 & 35 & 1 & 35 & 36 \\
\hline
\end{tabular}

of healthy volunteers had a presence of PSEN1c (Figure 3), but this polymorphism could not be assayed for 1 patient with psoriasis and three healthy controls, therefore those subjects were excluded from further statistical analysis. A difference in the prevalence of PSEN1a- $\left(\chi^{2}\right.$ mid$p$ value $=0.84)$, either PSEN1b- $\left(\chi^{2}\right.$ mid- $p$ value $\left.=0.57\right)$ and PSEN1c-positivity $\left(\chi^{2}\right.$ mid- $p$ value $\left.=0.219\right)$ between healthy controls and psoriatic patients was found to be non-significant (Tables 3, 4).

\section{Discussion}

$\gamma$-secretase is a transmembrane protease composed of four subunits: PSEN1, PSENEN, NCSTN and APH1A. The binding site of the substrate is on presenilin 1 near the active site. Nicastrin (NCSTN) with presenilin 1 (PSEN1) works mainly to stabilize the complex. The $\gamma$-secretase complex cleaves a series of transmembrane proteins, including Notch receptors and the amyloid precursor protein $[16,17]$. Regulating Notch signalization, it plays a key role in the differentiation of the epidermis and appendages of the skin, it also controls the growth and differentiation of keratinocytes. In the human epidermis, Notch1 is expressed in all the epidermal layers; Notch2 is expressed in the basal layer. In the mouse epidermis, however, Notch1 is expressed most strongly in the spinous layer, and Notch2 is also expressed in the spinous layer but not in the basal layer $[18,19]$. Notch ligands such as
Table 4. PSEN 1c

\begin{tabular}{|c|c|c|c|}
\hline \multirow[t]{2}{*}{ Study group } & \multicolumn{2}{|c|}{ PSEN1C } & \multirow[t]{2}{*}{ Total } \\
\hline & + & - & \\
\hline Psoriasis & 3 & 48 & 51 \\
\hline Control & 0 & 33 & 33 \\
\hline
\end{tabular}

Jagged and Delta-like family members are expressed in overlapping patterns with Notch in the epidermis of both humans and mice. Despite some differences in the expression pattern of Notch and its ligands, analyses using keratinocytes from human and mouse skin indicate that Notch signalling induces differentiation. Notch1 signalling in mouse keratinocytes stimulates expression of early differentiation markers such as keratin 1 and involucrin. This induction is consistent with the epidermal phenotype observed in mice that have constitutively activated Notch1 in the epidermis. Histologically, the Notch1 activated mice show acanthosis, corresponding to an increase in the differentiating (keratin1 positive) cell compartment of the epidermis. Conversely, a decrease in the differentiating cell compartment is observed in mice that have a keratinocyte specific deletion of the Notch1 gene. A substantial increase in the number of layers of proliferating cells occurs in the Notch1 deficient epidermis [20] (Figure 4).

The broad functions of Notch imply a tight connection between Notch signalling and the pathogenesis of skin diseases. Consistent with its role in promoting the differentiation of keratinocytes, the expression of Notch and its ligands is decreased in psoriasis, a chronic skin disease that is characterized by hyperproliferation and aberrant differentiation of keratinocytes. Psoriasis is a complex disease that exhibits altered homeostasis of keratinocytes as well as infiltration of leukocytes. It remains to be definitively established whether altered
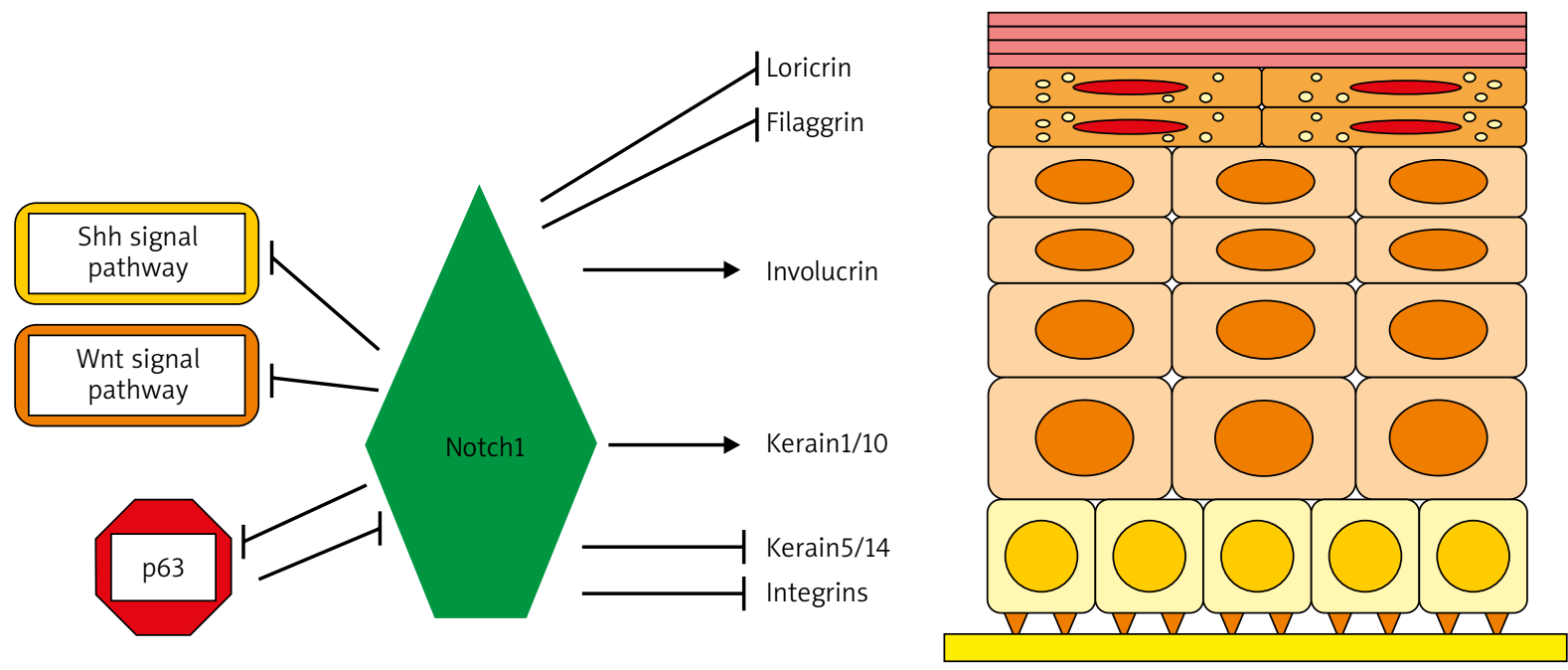

Figure 4. Notch1 function in epidermis (source: Okuyama R, Tagami H, Aiba S. Notch signalling: its role in epidermal homeostasis and in the pathogenesis of skin diseases. J Dermatol Sci 2008; 49: 187-94) 


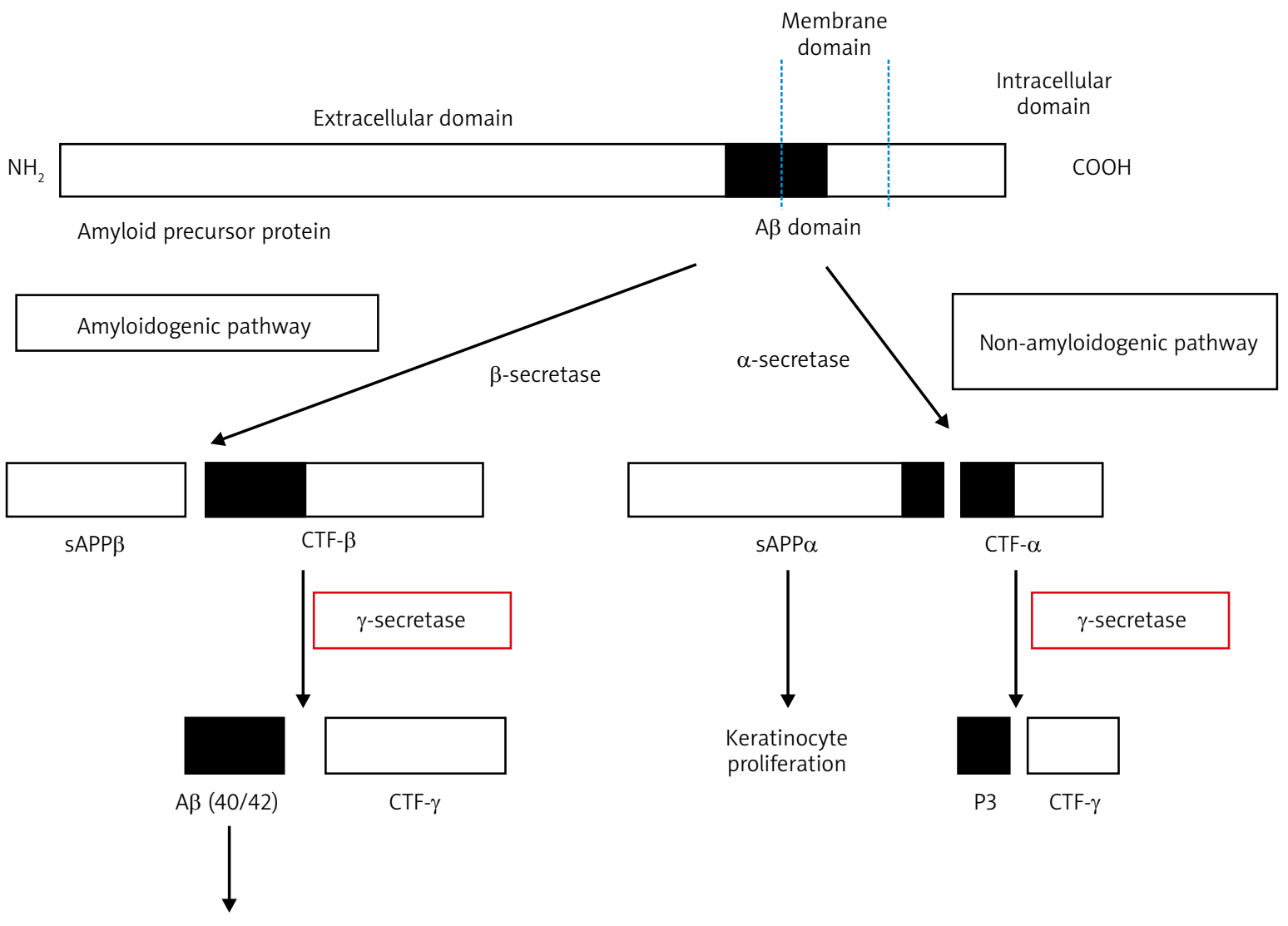

Alzheimer disease

$A \beta$ - amyloid- $\beta, C O O H$ - carboxylic acid, CTF - C-terminal fragment, $n b$-UVB - narrowband ultraviolet B therapy, NH2 - amine, P3 - non-amyloidogenic peptide, SAPP - soluble APP.

Figure 5. Pathways of processing of amyloid precursor protein (APP) by secretases [9]

Notch expression is a cause, rather than a consequence, of keratinocyte disturbance in psoriasis [21-26].

Mutations as well as polymorphisms of $\gamma$-secretase subunits have been confirmed in several disease entities, among others in the family form of acne inversa, Alzheimer's disease and dilated cardiomyopathy [14, 27]. In addition, a case of an exacerbation of psoriasis vulgaris was described in a woman who, due to Alzheimer's disease, received a $\gamma$-secretase inhibitor (LY450139) in clinical trials $[14,28]$. Hsu et al. [15] proposed 2 possible mechanisms for the exacerbation of psoriasis. Type 1 transmembrane proteins, such as Notch 1, may play a role as aberrant Notch signalling has been reported to be implicated in psoriasis. The other possible mechanism may be dysregulation of the soluble form of APP $\alpha$. In the non-amyloidogenic pathway, APP is cleaved by $\alpha$-secretase, releasing a large soluble fragment, soluble form of APP $\alpha$, and a membranebound C-terminal fragment (CTF)- $\alpha$. The CTF- $\alpha$ is then cleaved by $\gamma$-secretase to generate a $3-k d$ protein and CTF- $\gamma$. Inhibition of $\gamma$-secretase might result in the accumulation of the soluble form of APP $\alpha$, which is known to play a role in regulating the proliferation of normal and psoriatic keratinocytes. In the phase II study of LY450139
- of $\gamma$-secretase inhibitor, there were 3 cases of "possible drug-related" rashes manifesting as a diffuse macular rash - psoriasis-like, on the extremities and trunk (Figure 5).

This gives the premise that $\gamma$-secretase may be involved in the pathomechanism of ordinary psoriasis and wanting to get to know it more closely, we decided to investigate three PSEN1 subunit polymorphisms. What is more, there are not many reports on this subject in the literature. Examining 52 patients with psoriasis, only 2 of them had PSEN1a polymorphisms, three PSEN1b polymorphisms and three PSEN1c polymorphisms which did not give a statistically significant result compared to the control group. This study did not meet our expectations regarding the important role of the PSEN1 $\gamma$-secretase subunit in psoriasis vulgaris. The limitation of the work is the evaluation of only one gamma-secretase subunit. Further research is needed in this direction and now we start the assessment the other subunits of the $\gamma$-secretase gene PSENEN and NCSTN.

\section{Acknowledgments}

This study was sponsored by grant KNW-1-068/N/7/K. 


\section{Conflict of interest}

The authors declare no conflict of interest.

\section{References}

1. Szepietowski J, Adamski Z, Chodorowska G, et al. Diagnostics and treatment of psoriasis vulgaris: guidelines of the Polish Dermatological Society. Part I: mild psoriasis, psoriasis in children. Derm Rev 2012; 99: 83-96.

2. Harden JL, Krueger JG, Bowcock A. The immunogenetics of psoriasis: a comprehensive review. J Autoimmun 2015; 64: 66-73.

3. Ozawa A, Miyahara M, Sugai M, et al. HLA class I and II alleles and susceptibility to generalized pustular psoriasis: significant associations with HLA-CW1 and HLA-DQB1*0303. J Dermatol 1998; 25: 573-81.

4. Siemes C, Quast T, Klein E, et al. Normalized proliferation of normal and psoriatic keratinocytes by suppression of sAPPalpha-release. J Invest Dermatol 2004; 123: 556-63.

5. Bigler J, Rand HA, Kerkof K, et al. Cross-study homogeneity of psoriasis gene expression in skin across a large expression range. PLoS One 2013; 8: e52242.

6. Ruissen FV, Jansen BJH, De Jongh GJ, et al. A partial transcriptome of human epidermis. Genomics 2002; 79: 671-8.

7. Zhou X, Krueger J G, Kao MCJ, et al. Novel mechanisms of T-cell and dendritic cell activation revealed by profiling of psoriasis on the 63,100-element oligonucleotide array. Physiol Genomics 2003; 13: 69-78.

8. Itoh K, Kawasaki S, Kawamoto S, et al. Identification of differentially expressed genes in psoriasis using expression profiling approaches. Exp Dermatol 2005; 14: 667-74.

9. Okuyama R, Tagami H, Aiba S. Notch signaling: its role in epidermal homeostasis and in the pathogenesis of skin diseases. J Dermatol Sci 2008; 493: 187-94.

10. Kelleher RJ, Shen J. $\gamma$-Secretase and human disease. The role of an enzyme in disease pathogenesis extends beyond Alzheimer's disease to a skin disorder. Science 2010; 330: 1055-6.

11. Artavanis-Tsakonas S, Matsuno K, Fortini ME. Notch signaling. Science 1995; 268: 225-32.

12. Zhang C, Wu B, Beglapulos V, et al. Presenilins are essential for regulating neurotransmitter release. Nature 2009; 460: 632-6.

13. Shah A, Alhusayen R, Amini-Nik S. The critical role of macrophages in the pathogenesis of hidradenitis suppurativa. Inflamm Res 2014; 66: 931-45.

14. Wang B, Yang W, Wen W, et al. Gamma-secretase genes mutations in familial acne inversa. Science 2010; 330: 1065.

15. Hsu CK, Hsu CC, Lee JY, et al. Exacerbation of psoriatic skin lesions in a patient with Alzheimer disease receiving gamma-secretase inhibitor. J Am Acad Dermatol 2013; 68: e46-78.

16. Shutter JR, Scully S, Fan W, et al. Dll4, a novel Notch ligand expressed in arterial endothelium. Genes Dev 2000; 14: 1313-8.

17. Saxena MT, Schroeter EH, Mumm JS, et al. Murine notch homologs (N1-4) undergo presenilin-dependent proteolysis J Biol Chem 2001; 276: 40268-73.

18. Chiba S. Notch signaling in stem cell systems. Stem Cells 2004; 24: 2437-47.

19. Blanpain C, Lowry WE, Pasolli HA, et al. Canonical notch signaling functions as a commitment switch in the epidermal lineage. Genes Dev 2006; 20: 3022-35.
20. Okuyama R, Nguyen BC, Talora C, et al. High commitment of embryonic keratinocytes to terminal differentiation through a Notch1-caspase 3 regulatory mechanism. Dev Cell 2004; 6: 551-62.

21. Lowell S, Jones P, Le Roux I, et al. Stimulation of human epidermal differentiation by delta-notch signalling at the boundaries of stem-cell clusters. Curr Biol 2000; 10: 491-500.

22. Rangarajan A, Talora C, Okuyama R, et al. Notch signaling is a direct determinant of keratinocyte growth arrest and entry into differentiation. EMBO J 2001; 20: 3427-36.

23. Kageyama R, Ohtsuka T, Hatakeyama J, et al. Roles of bHLH genes in neural stem cell differentiation. Exp Cell Res 2005; 306: 343-8.

24. Radtke F, Raj K. The role of Notch in tumorigenesis: oncogene or tumour suppressor? Nat Rev Cancer 2003; 3: 756-67.

25. Nguyen BC, Lefort K, Mandinova A, et al. Cross-regulation between Notch and p63 in keratinocyte commitment to differentiation. Genes Dev 2006; 20: 1028-42.

26. Pan Y, Lin MH, Tian X, et al. Gamma-secretase functions through Notch signaling to maintain skin appendages but is not required for their patterning or initial morphogenesis. Dev Cell 2004; 7: 731-43.

27. Thelu J, Rossio P, Favier B. Notch signalling is linked to epidermal cell differentiation level in basal cell carcinoma, psoriasis and wound healing. BMC Dermatol 2002; 2: 7.

28. Trechot P, Schmutz JL. Exacerbation of psoriasiform lesions by a gamma-secretase inhibitor. Ann Dermatol Venereol 2013; 140: 669-70. 